\title{
Energy harvesting from passing train as source of energy for autonomous trackside objects
}

\author{
Zdenek Hadas*, Jan Smilek, and Ondrej Rubes \\ Faculty of Mechanical Engineering, Brno University of Technology, Technicka 2896/2, 61669 Brno, \\ Czech Republic
}

\begin{abstract}
This paper deals with an energy harvesting review and analysis of an ambient mechanical energy on a trackside during a passing of a train. Trains provide very high level of vibration and deformation which could be converted into useful electricity. Due to maintenance and safety reasons a rail trackside includes sensing systems and number of sensor nodes is increased for modern transportation. Recent development of modern communication and ultra-low power electronics allows to use energy harvesting systems as autonomous source of electrical energy for these trackside objects. Main aim of this paper is model-based design of proposed vibration energy harvesting systems inside sleeper and predict harvested power during the train passing. Measurements of passing train is used as input for simulation models and harvested power is calculated. This simulation of proposed energy harvesting device is very useful for future design.
\end{abstract}

\section{Introduction}

Evolution on train monitoring and signalling needs to be accompanied by an investigation of new technologies to be applied in the rail environment. Energy harvesting technologies could provide autonomous source of energy for several smart and ultra-low power trackside objects [1] which could monitor and communicate autonomously, and it provide efficient increasing of transport capacity. Main aim of this paper is summarizing of potential autonomous sources of electrical energy for rail transportation [2], mainly for smart low power objects on trackside environment.

The trackside environment provides several potential principles of energy harvesting from mechanical energy in a form of mechanical vibration, rail deformation and sleeper or rail sag, etc. Individual energy harvesting principles are summarized and assessed. A physical principle of vibration energy harvesting solution is proposed under this paper due to maintenance free reasons. A simulation model of the proposed design was also created for analysis of output power under sleeper applications.

\footnotetext{
*Corresponding author: hadas@,fme.vutbr.cz
} 


\section{Analysis of mechanical energy harvesting sources on trackside during passing trains}

Potential trackside energy harvesting technologies are illustrated and summarized in Fig. 1. Commercial solar and wind power stations are common electrical energy solution for trackside system with power requirement in range of $10 \mathrm{~W}-10 \mathrm{~kW}$. However, there are several sources of mechanical energy which are forced by a passing train. These mechanical sources of energy could be very useful for autonomous powering of monitoring, sensing, signalling and communication objects in the trackside environment.

The passing train causes deformations, sag and oscillations of railway parts (e.g. sleepers and rail). Relative displacements of sleeper against ground [3] (type 1), rail sag against ground [4] (type 2), rail against sleeper [5] (type 3) and displacement of individual sleepers [6] (type 4) could be harvested using several principles of electromechanical linear generators. As well mechanical vibration of sleeper [7] (type 5) and rail [8] (type 6) could employ physical principles of vibration energy harvesting methods. The passing trains locally deform the rail and this deformation could be converted into electricity [9] (type 7) by piezoelectric layers. The passing wheel could also run over a flexible element which could transform kinetic or potential energy into electricity by linear generator [10] (type 8). Last option of illustrated energy harvesting systems could use a change of magnetic field by passing wheel [11] which is inducing electrical energy (type 9).

Energy harvesting device could use a form of physical principle of electro-mechanical conversion. Mainly electromagnetic and piezoelectric energy harvesting solution could be compared [12] with respect to a required output power. However magnetostriction materials [13] could be used for type 3 too. Displacement energy harvesting devices have a potential to harvest electricity in range of tens watts but these devices require maintenance.

Potential trackside energy harvesting technologies:

- Commercial solar and wind power station

- Displacement energy harvesting - type 1,2,3,4

- Vibration energy harvesting - type 5,6

- Strain and deformation energy harvesting - type 7 and 8

- Change of magnetic field by passing wheel -9

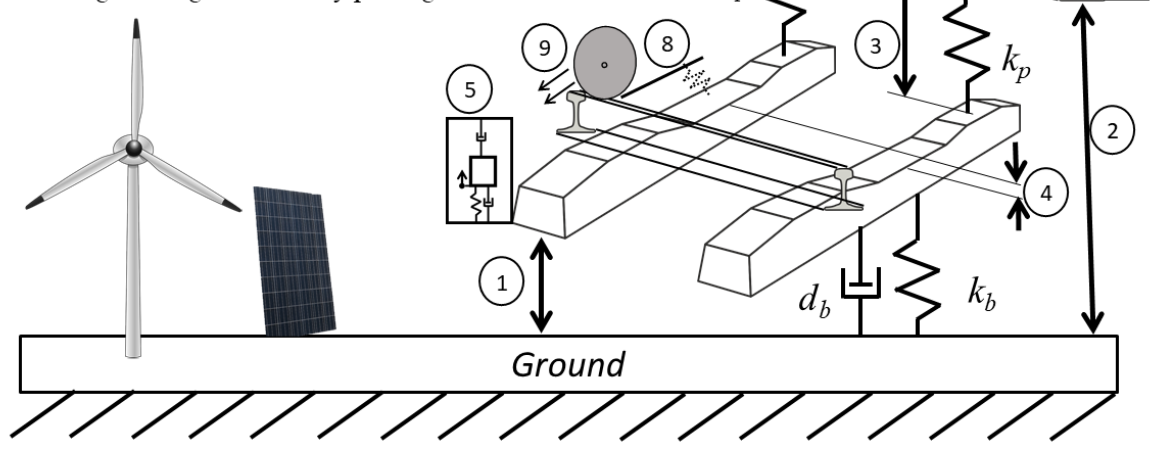

Fig. 1. Potential energy sources on trackside for energy harvesting.

\section{Vibration of sleeper as autonomous source of energy}

Vibration energy harvesting devices usually provide maintenance free design and these devices could be also integrated in parts of trackside (e.g. sleepers). However output power of these kinetic devices depends on a peak and width of acceleration pulses and frequency and it is usually very low (range of milliwatts) but it could be useful for new generation of ultra-low power and smart trackside objects for sensing and monitoring issues. 


\subsection{Model of kinetic electromagnetic energy harvester}

The passing train provides mechanical oscillation of the sleeper which depicted in Fig. 2. Sleeper oscillation is in form of sag pulses $x_{2}$ which depends on a train mass, velocity and dynamics of a rail bed (damping and stiffness of rail, sleeper suspension and ballast parameters). Due to acceleration pulses during the sag of sleeper this kinetic energy could be harvested using a resonator operation of a vibration energy harvesting system [14].

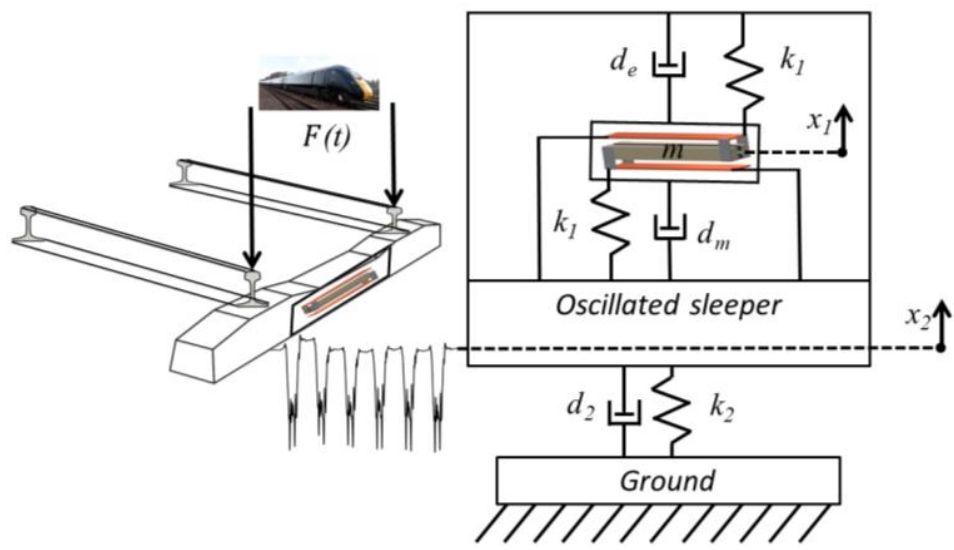

Fig. 2. Electromagnetic vibration energy harvesting device inside sleeper.

The proposed mechanical resonator is embedded inside a sleeper. The design is based on a two-cantilever suspension [15], stiffness $k_{l}$, of a moving seismic mass $m_{l}$. A frame of the resonator is anchored inside vibrating sleeper and therefore the mechanical resonator is excited by ambient mechanical shocks of the vibrating sleeper $x_{2}$ and it provides the relative movement $x_{I}$. The relative oscillation $x_{I}$ of the mass $m_{1}$ with magnetic circuit against a frame coil is found by solving the second order differential equation (1) and it is inversely proportional with mechanical damping $d_{m}$.

Due to Faraday's law the oscillation movement provides change of the magnetic field through the coil and it induces voltage on the coil. The voltage $u_{i}(2)$ depends on number of the coil turns $N$, active length of the coil $l$, magnetic flux density $B$ through active coil turns and on velocity of the relative movement from equation (1). The overall harvested electrical power is extracted from the system by an electromechanical transducer. The extraction provides an electromechanical damping effect (3), which is depicted as damper $d_{e}$ and where $Z$ is impedance of whole electrical circuit.

$$
\begin{gathered}
m_{1} \ddot{x}_{1}+d_{m} \dot{x}_{1}+d_{e} \dot{x}_{1}+2 k_{1} x_{1}=-m_{1} \ddot{x}_{2} \\
u_{i}=N \cdot l \cdot B \cdot \dot{x}_{1}(t) \\
d_{e}=\frac{(N \cdot l \cdot B)^{2}}{Z}
\end{gathered}
$$

\subsection{Experimental result of energy harvester under lab tests}

The presented vibration energy harvesting system operates very well with harmonic excitation but response of real operation on trackside must be analysed. Therefore an experimental test of a sensitive electromagnetic resonator, which is described in detail by publication [16], with operation frequency $17 \mathrm{~Hz}$ (Fig. 3a) was done in our vibration lab. This resonance energy harvester is also sensitive for mechanical shock and it was fixed on a 
lab shaker and this device was excited by an acceleration measurement of the real sleeper during several train passing. The harvester operation under the real measured excitation was observed and analysed for different resistive load and output voltage on the resistive load of $3 \mathrm{k} \Omega$ is shown in Fig. $3 \mathrm{~b}$ ). Maximal harvested power was calculated for several types of passing train and position of energy harvester on trackside. The presented results correspond with average output power $2 \mathrm{~mW}$ during train passing and it could be useful for several low power sensing and monitoring applications. However this vibration energy harvesting system could be redesigned for trackside applications and also resized. Than higher power outputs are expected and it has to be optimized [17] with respect to average speed of train and quality of trackside. This energy harvesting system could provide a maintenance free device and it could be integrated in new generation of sleeper.

a)

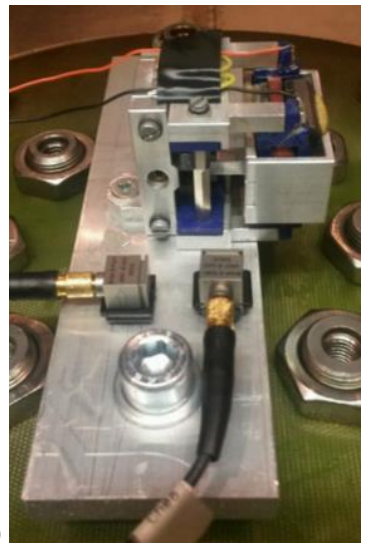

b)

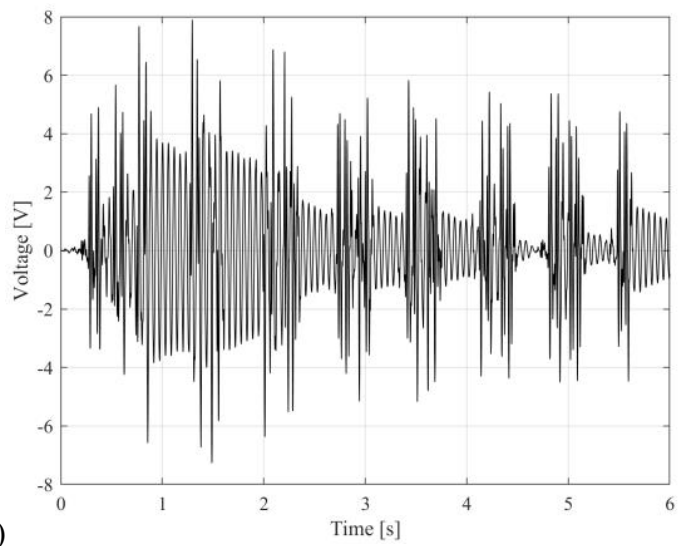

Fig. 3. a) Tested electromagnetic vibration energy harvester $17 \mathrm{~Hz}$; b) output voltage measurement during vibration excitation by measured vibration on sleeper - passing train with speed $130 \mathrm{~km} / \mathrm{h}$

\subsection{Set up of optimal operating frequency of kinetic energy harvester}

Tested energy harvester was designed for the resonance operation but the passing train provide pulse excitation and this fact has to be reflected for design of energy harvester.

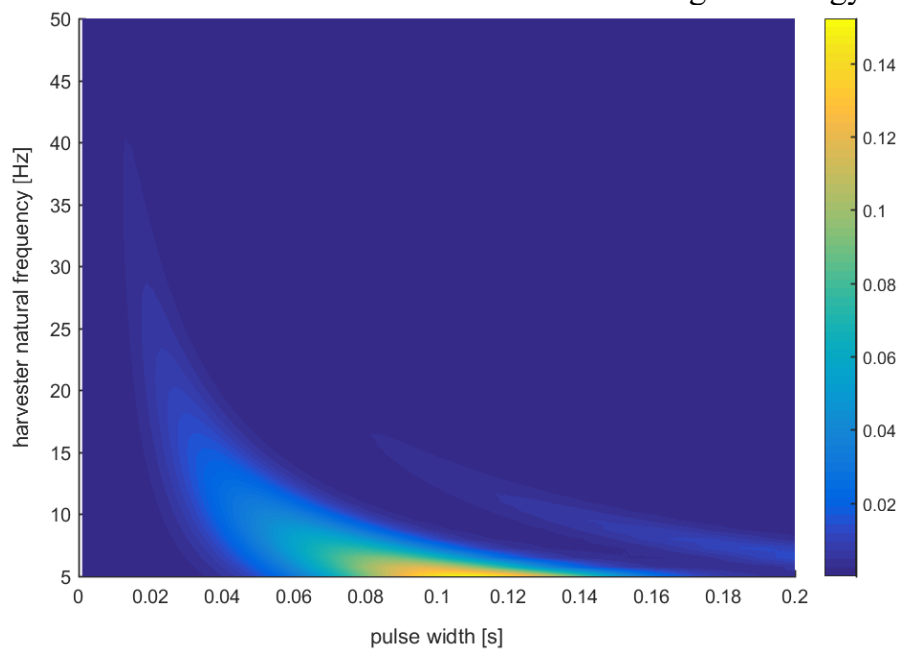

Fig. 4. Harvested energy [in Joules] from single $10 \mathrm{~m} \cdot \mathrm{s}^{-2}$ square acceleration pulse of variable width for different resonant frequencies of kinetic energy harvester 
Therefore a generic idealized linear simulation model (1) of kinetic energy harvester with constant electrical and mechanical damping factors and variable natural frequency was fed with triangular acceleration pulses of variable width to determine the optimal tuning of the harvester with respect to the total energy harvested from one set of triangular pulses (Fig. 4). One pulse set consists of three triangular pulses and represents passing of a single train wheel. The proof mass of the harvester was set to $1 \mathrm{~kg}(0.45$ is mass of FeNdB magnets), coil length is $800 \mathrm{~m}$ and the input acceleration peak was set to $1 \mathrm{~g}$ peak.

\subsection{Model-based design and simulation of passing train}

The simulation model [18] of vibration energy harvester (1) and (2) could be excited by an acceleration measured data from real oscillation of sleeper under the passing express train with speed $130 \mathrm{~km} / \mathrm{h}$. This train speed has maximal outputs for the operating frequency of kinetic energy harvester $32 \mathrm{~Hz}$ due to short width of acceleration pulses. Simulation results and outputs are shown in Fig. 5. The proposed energy harvesting device could harvest average output power around $200 \mathrm{~mW}$ during the measured train passing.
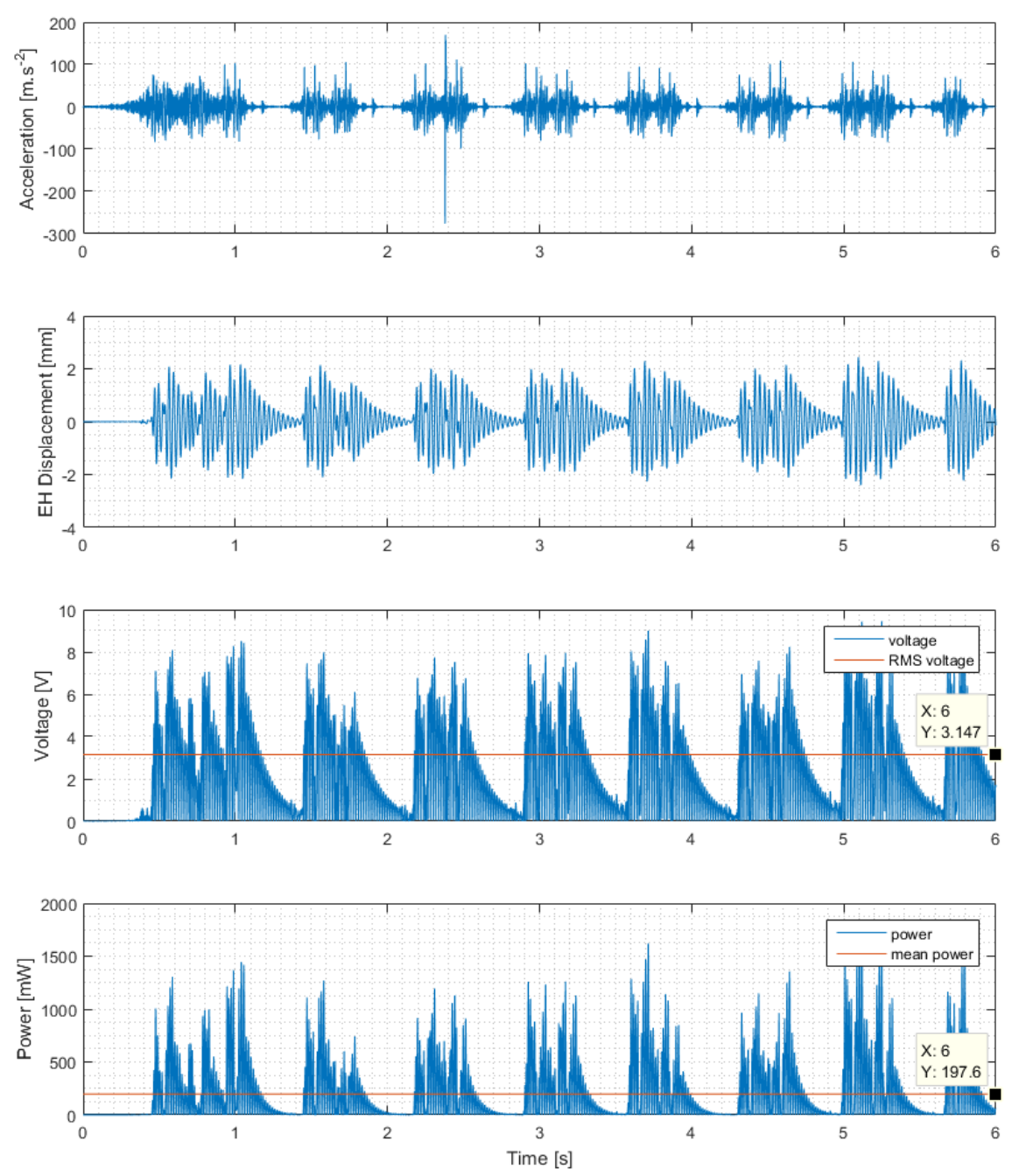

Fig. 5. Simulation of kinetic energy harvesting device inside switch sleeper 


\section{Conclusion}

The presented analysis, test and simulation of predicted harvested energy of the proposed kinetic energy harvesting device are very useful for development of the energy harvesting devices for future integration inside sleepers. This energy solution could provide the autonomous source of energy for smart trackside objects which could significantly improve safety, efficiency and capacity of train transportation. The proposed kinetic energy harvester will be developed under Shift-2-Rail project with title ETALON in 2019. On the base of predicted harvested energy from simulation and the communication duty cycle could be setup for future smart trackside objects. As well proposed approach could be useful for choice of suitable placement of these devices because quality of trackside is not the same in Europe and the trackside quality significantly affects harvested power and operation of autonomous objects.

This work is an output of research and scientific activities of NETME Centre, supported through project NETME CENTRE PLUS (LO1202) by financial means from the Ministry of Education, Youth and Sports „National Sustainability Programme I“.

\section{References}

[1] M. Gao, P. Wang, Y. Cao, R. Chen, and D. Cai, IEEE Trans. Intell. Transp. Syst. 1 (2016).

[2] X. Zhang, Z. Zhang, H. Pan, W. Salman, Y. Yuan, and Y. Liu, Energy Convers. Manag. 118, 287 (2016).

[3] Y. Tianchen, Y. Jian, S. Ruigang, and L. Xiaowei, Smart Mater. Struct. 23, 125046 (2014).

[4] X. Zhang, H. Pan, L. Qi, Z. Zhang, Y. Yuan, and Y. Liu, Appl. Energy 204, 1535 (2017).

[5] W. Wang, R.-J. Huang, C.-J. Huang, and L.-F. Li, Acta Mech. Sin. 30, 884 (2014).

[6] J. J. Wang, G. P. Penamalli, and L. Zuo, Proc. 2012 8th IEEE/ASME Int. Conf. Mechatron. Embed. Syst. Appl. MESA 2012 11787, 29 (2012).

[7] V. G. Cleante, M. J. Brennan, G. Gatti, and D. J. Thompson, J. Phys. Conf. Ser. 744, 12080 (2016).

[8] M. Gao, P. Wang, Y. Wang, and L. Yao, IEEE Trans. Intell. Transp. Syst. 1 (2017).

[9] J. Wang, Z. Shi, H. Xiang, and G. Song, Smart Mater. Struct. 24, 105017 (2015).

[10] A. Pourghodrat, C. A. Nelson, S. E. Hansen, V. Kamarajugadda, and S. R. Platt, Proc. Inst. Mech. Eng. Part F J. Rail Rapid Transit 228, 504 (2014).

[11] M. Kroener, S. K. T. Ravindran, and P. Woias, J. Phys. Conf. Ser. 476, 12091 (2013).

[12] Z. Hadas, J. Smilek, and O. Rubes, in Smart Sensors, Actuators, MEMS VIII, edited by

L. Fonseca, M. Prunnila, and E. Peiner (Barcelona, Spain, 2017), p. 1024619.

[13] J. Kaleta, K. Kot, R. Mech, and P. Wiewiorski, Key Eng. Mater. 598, 75 (2014).

[14] G. Gatti, M. J. Brennan, M. G. Tehrani, and D. J. Thompson, Mech. Syst. Signal Process. 66-67, 785 (2016).

[15] Z. Hadas and F. Ksica, in Proc. 16th Int. Conf. Mechatronics - Mechatronika 2014 (2014), pp. 393-398.

[16] Z. Hadas, V. Vetiska, V. Singule, O. Andrs, J. Kovar, and J. Vetiska, Int. J. Adv. Robot. Syst. 9, 1 (2012).

[17] Z. Hadas, J. Kurfurst, C. Ondrusek, and V. Singule, Microsyst. Technol. 18, 1003 (2012).

[18] Z. Hadas, V. Vetiska, R. Huzlik, and V. Singule, Microsyst. Technol. 20, 831 (2014). 